\title{
GEOMETRY OF DYNAMICAL TRAJECTORIES AT A POINT OF EQUILIBRIUM*
}

BY

EDWARD KASNER AND AARON FIALKOW $\dagger$

1. Introduction. A plane positional field of force gives rise to a threeparameter family of curves. These are the totality of possible paths or trajectories of a particle moving under the influence of the field. This system of curves is not arbitrary but has intrinsic peculiarities. Kasner's $\ddagger$ investigations of the geometry of this situation showed that many aspects of dynamics may be described in geometric language. Excluding points where the force vanishes, he obtained a set of five properties which completely characterize the system of trajectories. Further observations on these five properties are given in recent papers by Moisseiev $\S$ together with new kinematic properties.

In this note, we derive some similar geometric properties of the paths along which a particle may move when it is projected from an isolated point of equilibrium. The new properties are stated in terms of osculating cubics and higher algebraic curves. Since such a point is a singular point of the lines of force, the geometric character of the field is quite different from that at regular points. The qualitative nature of the lines of force follows, of course, from the classic work of Poincaré,\| Bendixson, $\uparrow$ and others on the singular points of ordinary differential equations of the first order. A few elementary connections between the differential geometry of the paths and the general character of the lines of force are given in this paper.

2. The equation of the trajectory. In what follows we assume that the force is analytic in the neighborhood of the point of equilibrium which we choose as the origin of coordinates. The components of the force are $\phi(x, y)$ and $\psi(x, y)$, where

$$
\begin{aligned}
& \phi(x, y)=A x+B y+G_{20} x^{2}+G_{11} x y+G_{02} y^{2}+\cdots, \\
& \psi(x, y)=C x+D y+H_{20} x^{2}+H_{11} x y+H_{02} y^{2}+\cdots .
\end{aligned}
$$

A particle of unit mass projected from the origin travels along a path whose parametric equations, in terms of the time $t$, are

* Presented to the Society, October 31, 1936; received by the editors October 17, 1936.

$\dagger$ National Research Fellow.

$\ddagger$ Differential-Geometric Aspects of Dynamics, American Mathematical Society Colloquium Publications, vol. 3, part 2, 1913; new edition, 1934. Also these Transactions, vol. 7 (1906), pp. 401-424.

§ Proceedings of the Sternberg Astronomical Observatory, vol. 7 (1936).

|l Journal de Mathématiques (1881, 1882); also other papers collected in Oeuvres, vol. 1, 1928.

I Acta Mathematica, vol. 24 (1901), pp. 1-88. 


$$
\begin{aligned}
& x=a_{1} t+a_{2} t^{2}+a_{3} t^{3}+\cdots, \\
& y=b_{1} t+b_{2} t^{2}+b_{3} t^{3}+\cdots .
\end{aligned}
$$

These equations are a solution of

$$
\begin{aligned}
& \frac{d^{2} x}{d t^{2}}=\phi(x, y), \\
& \frac{d^{2} y}{d t^{2}}=\psi(x, y) .
\end{aligned}
$$

To determine the $a_{i}$ and $b_{i}$, we substitute the values for $x$ and $y$ given by (2) in (1) and (3) and equate like powers of $t$. The first few equations resulting are

$$
\begin{aligned}
a_{2} & =0, \\
3 \cdot 2 \cdot a_{3} & =A a_{1}+B b_{1}, \\
4 \cdot 3 \cdot a_{4} & =G_{20} a_{1}{ }^{2}+G_{11} a_{1} b_{1}+G_{02} b_{1}{ }^{2} \\
5 \cdot 4 \cdot a_{5} & =A a_{3}+B b_{3}+G_{30} a_{1}^{3}+G_{21} a_{1}{ }^{2} b_{1}+G_{12} a_{1} b_{1}{ }^{2}+G_{03} b_{1} .
\end{aligned}
$$

Similar expressions obtain for the $b_{i}$. Since $a_{1}$ and $b_{1}$ are the components of the initial velocity, they are arbitrary parameters. For convenience we write* $m=b_{1} / a_{1}$ and $c_{i}=b_{i} a_{1}-a_{i} b_{1}$. If in $\phi(x, y)$ and $\psi(x, y)$, the terms of the $i$ th degree are $X_{i}(x, y)$ and $Y_{i}(x, y)$ respectively, we denote by $K_{i+1}(m)$ the polynomial $Y_{i}(1, m)-m X_{i}(1, m)$ of degree $(i+1)$ in $m$. From (4),

$$
\begin{aligned}
a_{2} & =b_{2}=0, \quad c_{2}=0, \\
3 \cdot 2 \cdot c_{3} & =a_{1}{ }^{2} K_{2}(m), \\
4 \cdot 3 \cdot c_{4} & =a_{1}{ }^{3} K_{3}(m), \\
5 \cdot 4 \cdot c_{5} & =a_{1}{ }^{4} K_{4}(m)+\frac{(A+D)}{3 \cdot 2} a_{1}{ }^{2} K_{2}(m) .
\end{aligned}
$$

If we eliminate $t$ between the two equations of (2), the cartesian equation of the path is obtained. $\dagger$ It is

* We assume that neither $a_{1}$ nor $b_{1}$ is zero in the derivation of (7) and (8). If $b_{1}=0$, it can be shown that the equations of the paths are (7) or (8) with $m=0$. If $a_{1}=0$, an analogous statement is true if we interchange $x$ and $y$ as well as $\phi$ and $\psi$.

$\dagger$ In Kasner's Transactions (1906) paper, it is shown that all the paths are the solutions of the third-order differential equation

$$
\left(\psi-\frac{d y}{d x} \cdot \phi\right) \frac{d^{3} y}{d x^{3}}=\left\{\psi_{x}+\left(\psi_{y}-\phi_{x}\right) \frac{d y}{d x}-\phi_{y}\left(\frac{d y}{d x}\right)^{2}\right\} \frac{d^{2} y}{d x^{2}}-3 \phi\left(\frac{d^{2} y}{d x^{2}}\right)^{2} .
$$

The coefficient of $d^{3} y / d x^{3}$ vanishes at a point of equilibrium. The equations (7) and (8) could also be obtained from this equation. 


$$
\frac{y}{b_{1}}=\frac{x}{a_{1}}+\frac{c_{3}}{a_{1} b_{1}}\left(\frac{x}{a_{1}}\right)^{3}+\frac{c_{4}}{a_{1} b_{1}}\left(\frac{x}{a_{1}}\right)^{4}+\frac{a_{1} c_{5}-3 a_{3} c_{3}}{a_{1}^{2} b_{1}}\left(\frac{x}{a_{1}}\right)^{5}+\cdots
$$

The values of $a_{i}, b_{i}$, and $c_{i}$ given by (4) and (5) are substituted in (6). The equation of the path becomes

$$
\begin{aligned}
y=m x & +\frac{K_{2}(m)}{3 \cdot 2 \cdot a_{1}{ }^{2}} x^{3}+\frac{K_{3}(m)}{4 \cdot 3 \cdot a_{1}{ }^{2}} x^{4} \\
& +\frac{1}{5 \cdot 4 \cdot a_{1}{ }^{2}}\left(K_{4}(m)+\frac{(D-9 A-10 B m) K_{2}(m)}{3 \cdot 2 \cdot a_{1}{ }^{2}}\right) x^{5}+\cdots .
\end{aligned}
$$

If $K_{2}(m), K_{3}(m), \cdots, K_{n-2}(m)$ vanish identically the coefficients of $x^{3}, x^{4}, \cdots, x^{n-1}$ are zero. The same is true if these polynomials are zero for a particular value of $m$. In both of these cases, after a complicated discussion we find that the equation of any trajectory having an initial slope $m$ for which $K_{2}(m), K_{3}(m), \cdots, K_{n-2}(m)$ but not $K_{n-1}(m)$ vanish, is

$$
\begin{aligned}
y=m x+ & \frac{K_{n-1}(m)}{n(n-1) a_{1}^{2}} x^{n}+\frac{K_{n}(m)}{n(n+1) a_{1}{ }^{2}} x^{n+1} \\
+ & \frac{1}{(n+1)(n+2) a_{1}^{2}} \\
& \left(K_{n+1}(m)+\frac{\left(D-n^{2} A-\left(1+n^{2}\right) B m\right) K_{n-1}(m)}{n(n-1) a_{1}{ }^{2}}\right) x^{n+2}+\cdots .
\end{aligned}
$$

From (7) and (8) it follows that the initial departures from their common tangent of all trajectories projected in the same direction vary inversely as the squares of the initial speeds. In this respect the behavior of the paths is the same at points of equilibrium as it is at regular points. We introduce the intrinsic measure $J$, defined as the first non-vanishing derivative of the curvature with respect to arc.* We thus have

THEOREM I. If particles are projected in the same direction from a point in a field of force, the quantities $J$ defined for their paths always vary inversely as the squares of the initial speeds.

Bendixson $\dagger$ studied the qualitative nature of the lines of force near an

* In previous papers, Kasner used the symbol $J$ (which he termed "inflexure") for the value of the derivative of the curvature of a curve with respect to its arc length at a point of inflexion. It is the invariant measure of the curve at its inflexions analogous to curvature at ordinary points. In this paper, we use this same symbol for the first non-vanishing derivative of the curvature with respect to arc length. Thus $J$ is defined for all analytic curves except straight lines.

$\dagger$ For a proof of his theorems which are used below as well as a precise statement of the conditions under which they are true, see Bendixson, loc. cit., pp. 34, 36, and 62. 
isolated singular point of the field. In this paper we are considering the differential-geometric character of the paths. In what follows we.indicate certain connections between these two aspects of the field of force.

It was shown by Bendixson that if $X_{i}(x, y) \equiv Y_{i}(x, y) \equiv 0,(i=1,2, \cdots$, $n-3)$, then only the real roots of $K_{n-1}(m)=0$ may be and, in general, are the initial directions of the lines of force which approach the point of equilibrium. If $K_{n-1}(m)$ is identically zero, then every ray from the point (except, possibly, for a finite number of singular rays) is the initial tangent of one and only one line of force. If $K_{n-1}(m)=0$ has no real roots, he showed that the lines of force are either spirals or closed curves about the point. These results of Bendixson together with equation (8) give

THEOREM II. If particles are projected in different directions from a point of equilibrium, then, in general, any path which is initially tangent to any line of force has higher order of contact with its tangent than a path which is not tangent to a line of force.

THEOREM III. If all trajectories oblained by projection from a point of equilibrium have the same order of contact with their tangents, then either there exists a line of force which approaches the point of equilibrium along each ray (except, possibly, for a finite number of rays), or every nearby line of force is either a spiral or a closed curve about the point.

3. Geometric properties for the simplest case. We proceed to translate equations (7) and (8) into geometric language. As is obvious from (7), every trajectory through the origin has at least second order contact with its tangent. At a regular point, on the contrary, the path has a point of inflexion only if the particle is projected in the direction of the force.*

In the simplest case, $X_{1}(x, y), Y_{1}(x, y)$, and $K_{2}(m)$ do not vanish identically. Then, at the origin, from (7),

$$
\begin{aligned}
& \frac{d^{2} y}{d x^{2}}=0, \\
& \frac{d^{4} y}{d x^{4}}=\frac{2 K_{3}(m)}{K_{2}(m)} \cdot \frac{d^{3} y}{d x^{3}} .
\end{aligned}
$$

According to (9), a curve which osculates a trajectory at the origin must have an inflexion there. We cannot therefore use parabolas as in the ordinary case. The simplest curves with inflexions are cubic curves. We shall restate (9) and (10) as theorems about certain cubic curves which have fourth order contact with the trajectories. The curves that we choose are the cubics which

\footnotetext{
* Kasner, Proceedings of the National Academy of Sciences, vol. 20 (1934), p. 131, Theorem 3.
} 
have a double point at infinity at which the line at infinity is one of the tangents. Of course, the other tangent is the asymptote of the cubic. We call each of these curves a "special cubic."

In order to simplify the calculations which follow, we first suppose $m=0$. When we take account of (9), the special cubic becomes

$$
(y-a x)^{3}+b y(y-a x)+c y=0,
$$

where $a$ is the slope of the asymptote and $c$ is a non-zero constant.* By a rotation of axes through an angle $\theta$, where $\tan \theta=m$, we obtain the general equation of the special cubic as

$$
\begin{aligned}
& \cos ^{3} \theta[(1-a m) y-(a+m) x]^{3} \\
& \quad+b \cos ^{2} \theta\left[m(m+a) x^{2}+\left(a m^{2}-2 m-a\right) x y+(1-a m) y^{2}\right] \\
& \quad+c \cdot \cos \theta \cdot[y-m x]=0 .
\end{aligned}
$$

The asymptote of (11) is easily found to be

$$
y(1-a m)=(a+m) x-c\left(1+m^{2}\right)^{1 / 2} / b .
$$

From (11), by differentiation

$$
\begin{aligned}
& \frac{d^{3} y}{d x^{3}}=\frac{6 a^{3}\left(1+m^{2}\right)^{2}}{c}, \\
& \frac{d^{4} y}{d x^{4}}=\frac{4 a b\left(1+m^{2}\right)^{1 / 2}}{c} \cdot \frac{d^{3} y}{d x^{3}}
\end{aligned}
$$

at the origin. From the equations (10) and (13), it follows that $b / c=K_{3}(m)$ $/\left[2 a K_{2}(m)\left(1+m^{2}\right)^{1 / 2}\right]$. Substituting this value in (12), the equation of the asymptote becomes

$$
y-\frac{2 m K_{2}(m)}{K_{3}(m)}=\tan (\theta+\alpha)\left(x-\frac{2 K_{2}(m)}{K_{3}(m)}\right),
$$

where $\alpha=\operatorname{arc} \tan a$ and is the angle between the initial tangent and the asymptote. It follows from (14) that if $m$ is constant the asymptotes all intersect at a fixed point which is on the initial tangent, thus forming a pencil. This proves

THEOREM IV. The special cubics which, at a point of equilibrium, osculate the $\infty^{1}$ trajectories passing through the given point in the same direction have asymptotes which form a pencil whose center lies on the common initial tangent.

* The additional condition that $c$ is a non-zero constant may easily be stated in geometric language. In the general case which is discussed in $\$ 4$ below, similar restrictions are made. 
By reversing the argument given above it is easy to derive a converse statement. We consider any two-parameter family of curves, $\infty^{1}$ in each direction, each having an inflexion at the origin. If the asymptotes of the special cubics osculating those curves which have the same initial slope form a pencil with center on the common tangent, then the differential elements at the origin obey the equation

$$
\frac{d^{4} y}{d x^{4}}=f(m) \cdot \frac{d^{3} y}{d x^{3}}
$$

As $m$ varies, the center of the pencil describes a locus. The parametric equations of this locus, by (14), are

$$
x=\frac{2 K_{2}(m)}{K_{3}(m)}, \quad y=\frac{2 m K_{2}(m)}{K_{3}(m)} .
$$

Eliminating $m$, we find the cartesian equation of the locus. It is

$$
x K_{3}\left(\frac{y}{x}\right)-2 K_{2}\left(\frac{y}{x}\right)=0 .
$$

This is a cubic curve with a double point at the origin. This equation in conjunction with a theorem of Bendixson previously cited proves

THEOREM V. The locus of the centers of the $\infty^{1}$ pencils of asymptotes corresponding to a point of equilibrium is a cubic of which that point is a double point. The real semi-tangents at the double point are the only rays along which lines of force approach the point of equilibrium. If no real tangents exist, then all the nearby lines of force are either spirals or closed curves about the point.

Conversely, if the locus of the centers is any cubic curve having a double point at the origin, $f(m)$ in (15) must be a rational function of $m$ whose numerator and denominator are of the third and second degrees respectively.

4. Geometric properties for the general case. We now consider the case in which the leading polynomials present in $\phi(x, y)$ and $\psi(x, y)$ are $X_{p-2}(x, y)$ and $Y_{p-2}(x, y),(p \geqq 3)$, respectively.* If $K_{p-1}(m)$ is identically zero there is, in general, one line of force corresponding to each ray from the origin. If $K_{p-1}(m)$ does not vanish identically, the possible rays along which lines of force may approach the origin are given by the real roots of $K_{p-1}(m)=0$.

In the following we sketch the proofs of analogues of Theorems IV and V for the general case. Let $K_{n-1}(m),(n \geqq p)$, be the first $K_{i}(m)$ which is not identically zero. Then the paths are given by (8). At the origin

${ }^{*}$ If $\phi(x, y)$ and $\psi(x, y)$ do not have leading polynomials of the same degree it is always possible to obtain equivalent components of the force of the desired type by a suitable rotation of axes. 


$$
\begin{aligned}
\frac{d^{i} y}{d x^{i}} & =0 \quad(i=2,3, \cdots, n-1), \\
\frac{d^{n+1} y}{d x^{n+1}} & =\frac{(n-1) K_{n}(m)}{K_{n-1}(m)} \cdot \frac{d^{n} y}{d x^{n}} .
\end{aligned}
$$

As in $\S 3$, these equations may be converted into properties of osculating curves. The curves that we select have $(n+2)$ points in common with the trajectories at the origin. They are special $n$-ics which have a double point at infinity at which the line at infinity has contact of $(n-2)$ nd order with the $n$-ic. The other line having the same contact with the $n$-ic is its asymptote. Imposing conditions (16), the equation of the special $n$-ic is (11) after the exponent 3 is replaced by $n$. From (17) it follows by a method similar to that used in obtaining (14) that the asymptotes of those special $n$-ics which have the same $m$ form a pencil whose center is $\left(\left[(n+1) K_{n+1}(m)\right] /\left[(n-1) K_{n}(m)\right]\right.$, $\left.\left[(n+1) m K_{n-1}(m)\right] /\left[(n-1) K_{n}(m)\right]\right)$. We thus have

Theorem VI. The special $n$-ics which, at a point of equilibrium, osculate the $\infty^{1}$ trajectories passing through the point in the same direction have asymptotes which form a pencil whose center lies on the initial tangent.

As $m$ varies, the center of the pencil describes a locus whose equation is

$$
(n-1) x K_{n}\left(\frac{y}{x}\right)-(n+1) K_{n-1}\left(\frac{y}{x}\right)=0 .
$$

As in $\$ 3$, this proves

ThEOREM VII. The locus of the centers of the $\infty^{1}$ pencils of asymptotes corresponding to a point of equilibrium is an $n$-ic of which that point is an $(n-1)$ point. If $n=p$, the real semi-tangents at the $(n-1)$-point are the only rays along which lines of force may approach the point of equilibrium. If no real tangents exist, then all the nearby lines of force are either spirals or closed curves about the point. For $n>p$, there is, in general, one and only one line of force which approaches the point of equilibrium along each ray (except, possibly, for a finite number of rays).

A reversal of the argument employed to obtain these theorems yields converse statements similar to those of $\S 3$.

Columbia University,

New York, N. Y.

Princeton University and The Institute for Advanced Study,

Princeton, N. J. 